\title{
Sonographische Kontrolle des \\ Außenbandapparates am oberen \\ Sprunggelenk bei der frischen Bandruptur \\ und chronischen Bandinstabilität
}

\author{
R. Ernst ${ }^{l}, J . G^{2} i f k a^{2}$, R. Gritzan ${ }^{2}, M$. Kemen $^{\prime}, A$. Weber $^{3}$ \\ ${ }^{1}$ Chirurgische Klinik der Ruhr-Universität, St. Josef-Hospital, Bochum \\ (Direktor: Prof. Dr. med. V. Zumtobel) \\ ${ }^{2}$ Orthopädische Klinik der Ruhr-Universität, St. Josef-Hospital, Bochum \\ (Direktor: Prof. Dr. med. J. Krämer) \\ ${ }^{3}$ Radiologische Klinik der Ruhr-Universität, St. Josef-Hospital, Bochum \\ (Direktor: Prof. Dr. med. O. Spanke)
}

\section{Zusammenfassung}

Für die Instabilitätsdiagnostik der Außenbänder des oberen Sprunggelenkes wurde eine spezielle sonographische Untersuchungstechnik entwickelt. Bei 41 Patienten mit frischen Rupturen und 8 Patienten mit chronischen Instabilitäten wurde die sonographische Testung prospektiv präoperativ durchgeführt und mit dem Operationsbefund verglichen. Die Sensitivität von 0,96 für die Verletzungen des Lig. fib. tal. ant. und von 0,79 für das Lig. fib. calc. belegt die Sicherheit dieser Methode, die bei guter Patienten-Akzeptanz einfach durchzuführen und exakt zu dokumentieren ist.

\section{Sonographic examination of lateral ankle-joint ligaments with acute ruptures and chronic instability}

For diagnosis of instability in case of ruptures of the lateral ankle joint ligaments a special ultrasound examination technique was established. In 41 patients with acute ruptures and 8 patients with chronic instabilities a prospective preoperative sonographic instability-testing was performed and compared to intraoperative result. A sensitivity of 0.96 for injuries of the lig. fib. tal. ant. and 0.79 for the lig. fib. calc. proves the exactness of this methode. This technique is easy to perform and the result can be documented. This examination is accepted by the patients.

\section{Einleitung}

In der täglichen Praxis ist das Supinationstrauma des oberen Sprunggelenkes eine häufige Verletzung. 15-20\% der Sportverletzungen betreffen das obere Sprunggelenk, wobei Ballsportarten die höchste Verletzungsrate aufweisen $(5,10)$. Unzureichende Behandlung kann zur chronischen Bandinstabilität führen. Klinische

Z. Orthop. 128 (1990) 525-530

(C) 1990 F. Enke Verlag Stuttgart
Untersuchung und Anamnese führen meist zur richtigen Diagnose. Frakturen werden durch Röntgenaufnahmen in zwei Ebenen ausgeschlossen. „Gehaltenen Röntgenaufnahmen " haben sich zur Objektivierung und Dokumentation des Befundes durchgesetzt. Wir selbst verwenden dazu den Halteapparat nach Scheuba (3). Seit Anfang 1987 haben wir untersucht, ob mit Hilfe der Sonographie die Stabilität des Außenbandapparates des oberen Sprunggelenkes exakt geprüft werden kann.

Eine direkte Darstellung der Bandstrukturen und vor allem der Rupturstellen ist uns und anderen Autoren $(4,11)$ mit den heute zur Verfügung stehenden Ultraschallgeräten nicht sicher gelungen, so daß auf die Funktionsdiagnostik der Gelenkinstabilität zurückgegriffen werden muß. Wir haben dazu unsere eigene Methode entwickelt.

\section{Methode}

Wir schallen das Sprunggelenk senkrecht von dorsal in Längsachse des Unterschenkels über dem lateralen Anteil des tibio-talaren Gelenkspaltes. Der Patient liegt in Bauchlage, die Sprunggelenke nehmen bei leichter Beugung im Kniegelenk eine ca. 90-Grad-Stellung ein. Die schematische Darstellung der so definierten Untersuchungsschnittebene zeigt, daß aus derselben Ausgangsposition sowohl eine Beurteilung des Talusvorschubes als auch der Taluskippung möglich ist (Abb. 1 und 2).

Durch entsprechende Handgriffe wird unter „Realtime“-Bedingungen die Stabilität geprüft (Abb. 3). Die Änderung des Abstandes zwischen Tibiahinterkante und hinterer Begrenzung der Talusrolle ist das Maß für die Instabilität. Der Patient soll die Muskeln entspannen, Gewaltanwendung ist strikt zu vermeiden, da so provozierte Schmerzen sofort Gegenspannen bewirken und das Untersuchungsergebnis verfälschen. Standardisierte Belastung ist nicht erforderlich. Die Gegenseite wird stets mituntersucht. Die Dokumentation enthält die Abstandsmessungen.

Die neue Methode wurde in einer Pilotstudie an gesunden Probanden und Patienten mit klinisch und 

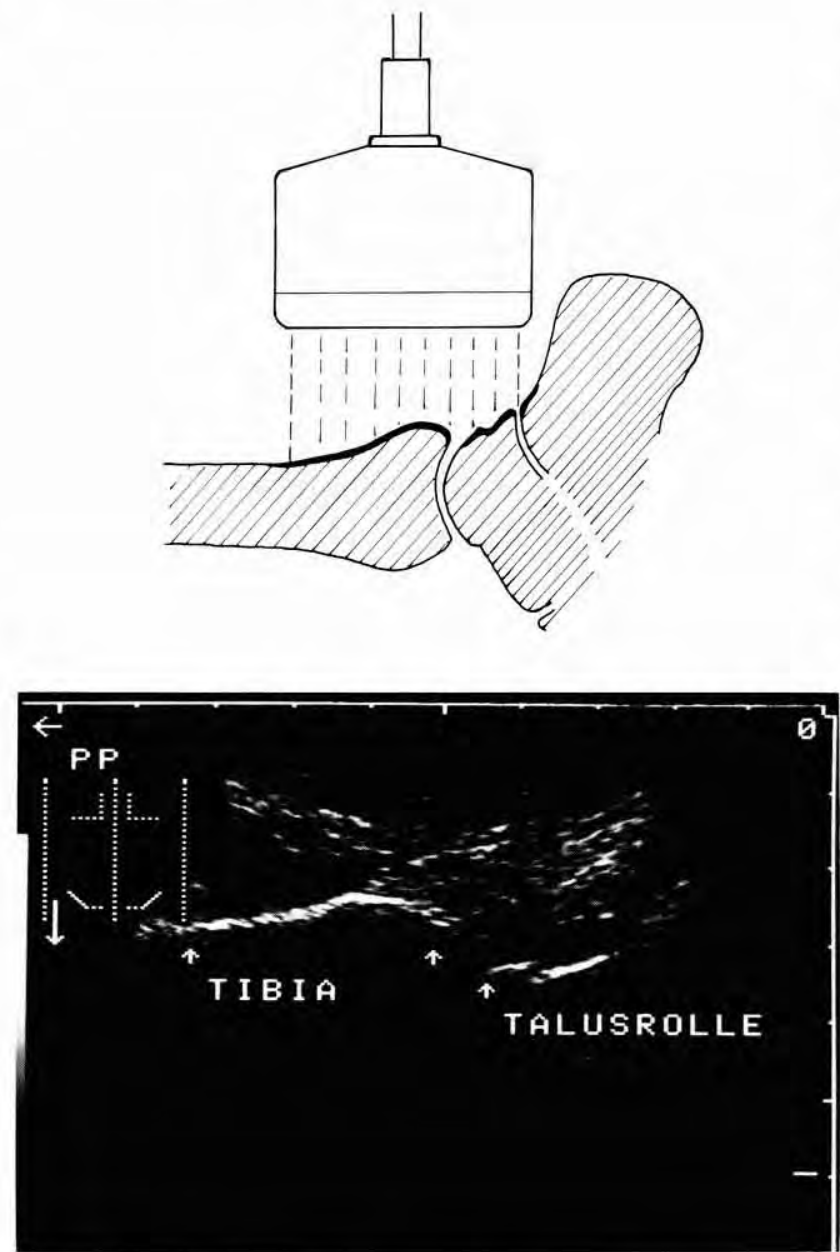

Abb. 1 Schematische Darstellung und Ultraschallbild der definierten Untersuchungsschnittebene in der Ausgangsposition für die Ultraschalluntersuchung:

a) Sagittaler Längsschnitt durch das obere Sprunggelenk in schematischer Darstellung. Die markante Kontur der Tibiahinterkante und der hinteren Begrenzung der Talusrolle ist hervorgehoben.

b) Wenn die Ausgangsposition exakt eingestellt ist, zeigt sich die gleiche markante Kontur im Ultraschallbild. Es muß auf eine klare Abbildung der Knochenstrukturen geachtet werden. Talusrolle und Tibiahinterkante sind mit Pfeilen markiert. radiologisch eindeutigen Verletzungen überprüft. In die anschließende prospektive Studie wurden nur Patienten aufgenommen, deren Sonographiebefund bei der Operation kontrolliert werden konnte. Die Operationsindikation wurde jeweils aufgrund des klinischen und radiologischen befundes gestellt. Es waren 41 Pat. mit frischen Bandrupturen und 8 Pat. mit chronischer Bandinstabilität. Das Durchschnittsalter betrug 23 Jahre.

Untersucht wurde mit einem $5 \mathrm{MHz}$-Realtime-Scanner, (meist Konvex-Scanner, Fa. Picker, LSC 7000). Eine Wasservorlaufstrecke war nicht erforderlich bei frischen Verletzungen mit Hämatom, ist jedoch hilfreich bei fehlender Schwellung, dünnen Weichteilen. Fußschalter zum „Einfrieren“ des Bildes ermöglichen die Bandprüfung durch einen einzigen Untersucher.

Für die exakte Dokumentation der OP-Befunde, die regelmäßige Revision des gesamten Außenbandapparates (Ligg. fibulo-talare anterius, fibulo-calcaneare und fibulo-talare posterius), der Syndesmose und anderer einsehbarer Strukturen zeichneten drei qualifizierte Operateure verantwortlich.

\section{Ergebnisse}

Bei 47 Patienten war das Ligamentum fibulo-talare anterius vollständig rupturiert. Zusätzlich hatten 27 dieser 47 Patienten eine komplette Ruptur des Ligamentum fibulo-calcaneare, 20 Patienten eine Teilruptur. Ein Patient hatte eine isolierte Teilruptur des Lig. fibulo-talare anterius. Bei einem Pat. zeigte sich eine Teilruptur des Lig. fibulo-talare anterius zusammen mit einer kompletten Ruptur des Lig. fibulo-calcaneare. Drei Patienten wiesen eine Teilruptur des Ligamentum fibulo-talare posterius auf, bei kompletter Ruptur der beiden anderen Bänder, die übrigen Fälle zeigten keine Verletzung des Lig. fibulo-talare posterius.

Ein Talusvorschub von $6 \mathrm{~mm}$ und mehr sowie eine Taluskippung von $6 \mathrm{~mm}$ und mehr erwies sich als pathologisch und beweisend für eine Bandverletzung, sofern die unverletzte Gegenseite keine Instabilität mit Bewe-

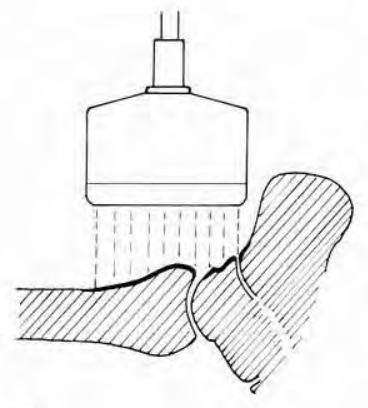

a)

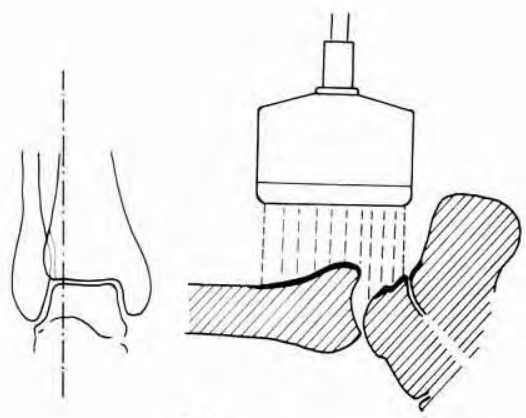

b)
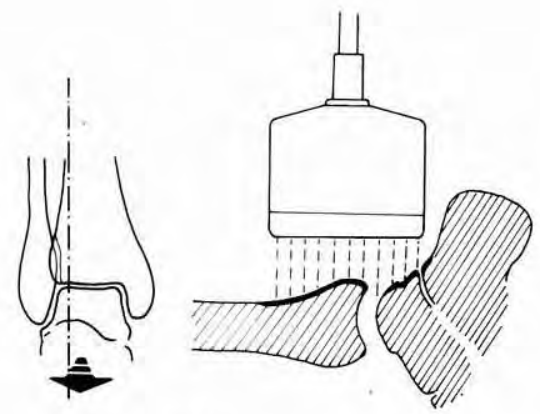

c)

Abb. 2 Schematische Darstellung der definierten Untersuchungsschnittebene während der Prüfung des Talusvorschubes und der Taluskippung

a) Die definierte Untersuchungsschnittebene in schematischer Darstellung. Sie erlaubt aus der Ausgangsposition sowohl eine Kontrolle des

b) Talusvorschubes als auch der

c) Taluskippung 


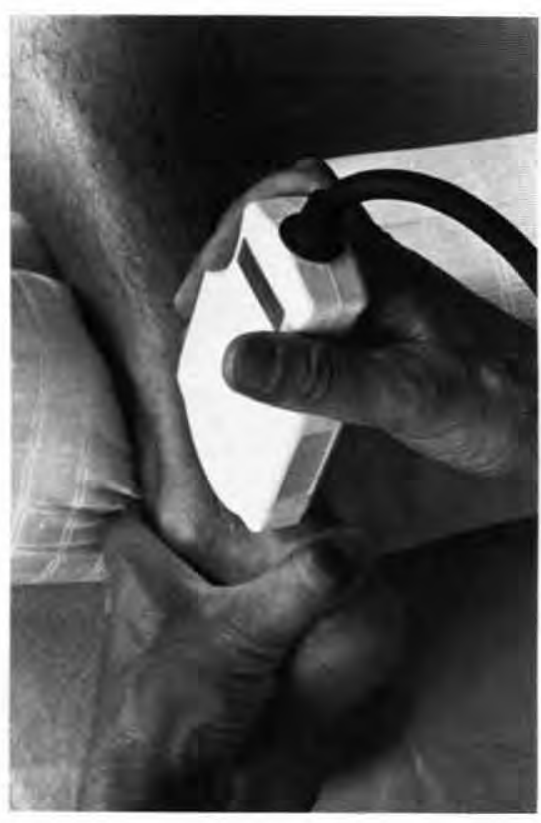

Abb. 3 Handgriffe zur Bandprüfung: Die eine Hand des Untersuchers fixiert den Schallkopf in der definierten Untersuchungsschnittebene. a) Prüfung des Talusvorschubes von dorsal gesehen: die Langfinger der anderen Hand stabilisieren die Tibia, der Daumen löst den Talusvorschub durch Druck auf den Calcaneus aus (Zangengriff).

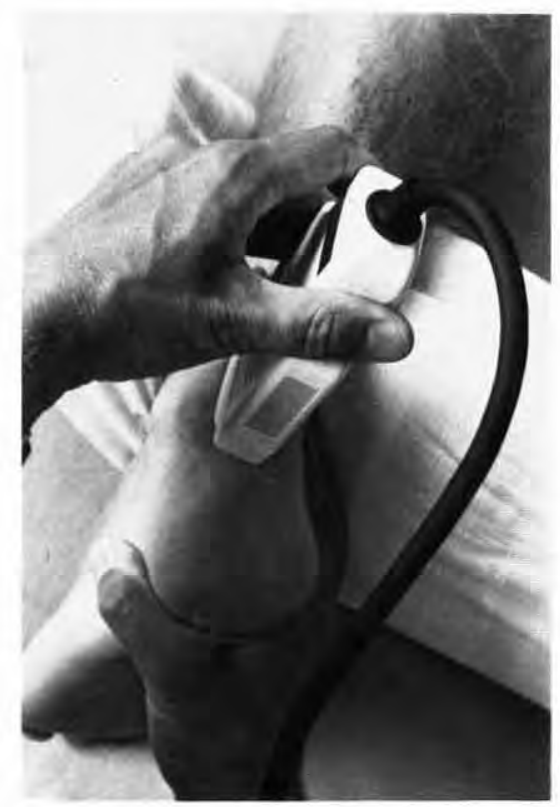

b) Ansicht von dorsal bei der Prüfung der Taluskippung: die zweite Hand des Untersuchers umfaßt die Ferse. Die Langfinger stützen sich am Innenknöchel bei der Stabilitätsprüfung $a b$.

gungsausschlag über $3 \mathrm{~mm}$ aufwies. Bei Ruptur des Ligamentum fibulo-talare anterius ohne Berücksichtigung des evtl. zusätzlichen Verletzungsmusters fanden wir einen Talusvorschub von $7,3 \pm 1,1 \mathrm{~mm}$ (Durchschnittswert mit Standardabweichung). Bei Ruptur des Ligamentum fibulo-calcaneare ohne Berücksichtigung des evtl. weiteren Verletzungsmusters ergab sich für die Taluskippung $6,3 \pm 1,0 \mathrm{~mm}$ (Durchschnittswert mit Standardabweichung). Bei kompletter Ruptur des Lig. fibulo-talare anterius und Teilruptur des Lig. fibulo-calcaneare fanden wir eine Taluskippung von 3,5 $\pm 0,9 \mathrm{~mm}$ (Durchschnitt mit Standardabweichung).

Betrachten wir den Talusvorschub nur als Maß für die Verletzung des Lig. fibulo-talare anterius und die Taluskippung nur als Maß für die Verletzung des Lig. fibulo-calcaneare, so ergibt sich folgende diagnostische Treffsicherheit der Sonographie (Tab. 1):
Tab. 1 Treffsicherheit der sonographischen Untersuchungstechnik bei Außenbandläsionen des oberen Sprunggelenkes in Abhängigkeit zu dem vorliegenden Verletzungsmuster, das bei der Operation festgestellt worden ist, bei 49 Patienten.

Der Talusvorschub ist nur für die Beurteilung des Ligamentum fibulo-talare anterius und die Taluskippung nur für die Beurteilung des Ligamentum fibulo-calcaneare herangezogen worden. (Abkürzungen:

Ligamentum fibulo-talare anterius $=$ ant.

Ligamentum fibulo-calcaneare $=$ calc.

Ligamentum fibulo-talare posterius $=$ post.)

Richtige Vorhersage der Verletzung durch Ultraschalluntersuchung (n von $n$ richtig)

\begin{tabular}{l|c|c|c}
$\begin{array}{l}\text { Verletzungsmuster } \\
\text { (Operationsbefund) }\end{array}$ & $n$ & $\begin{array}{l}\text { Talusvorschub } \\
\text { (Kontrolle: ant.) }\end{array}$ & $\begin{array}{l}\text { Taluskippung } \\
\text { (Kontrolle: calc.) }\end{array}$ \\
\hline $\begin{array}{l}\text { Ruptur ant. } \\
\text { Ruptur calc. }\end{array}$ & $3 / 3$ & 3/ 3 \\
Teilruptur post. & 3 & &
\end{tabular}

Ruptur ant.

Ruptur calc. post $0 . B$.

$24 / 24$

Ruptur ant.

Teilruptur calc. post o.B.

Teilruptur ant. Ruptur calc. post $0 . B$.

Teilruptur ant. calc. O.B. post. O.B 24

$20 / 20$ 20
0) 1\#

0) $1 \$$

Sämtliche Rupturen des Lig. fibulo-talare anterius wurden richtig diagnostiziert, beide Teilrupturen wurden als komplette Rupturen fehlgedeutet. Bei den Teilrupturen lag in einem Fall eine deutliche Überdehnung und Gelenkkapselruptur vor. Im zweiten Fall fanden wir neben der Teilruptur des Ligamentum fibulo-talare anterius zusätzlich eine komplette Ruptur des Ligamentum fibulo-calcaneare.

25 von 28 Rupturen des Lig. fibulo-calcaneare wurden richtig erkannt. Dreimal haben wir komplette Rupturen des Lig. fibulo-calcaneare als Teilrupturen fehlgedeutet. 13 von 20 Teilrupturen wurden richtig erkannt, vier als komplette Rupturen und drei als ohne Ruptur fehlgedeutet.

Rechnerisch ergibt sich somit für unsere Methode für die Beurteilung der Verletzung des Lig. fibu- 


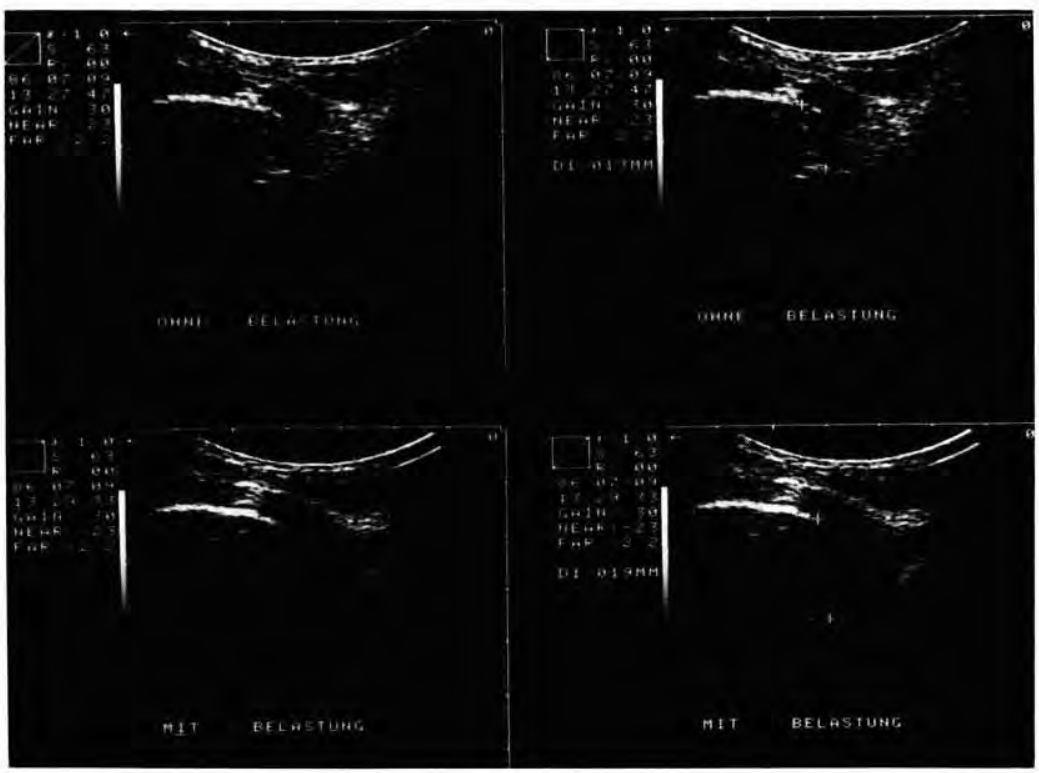

Abb. 4 Meßvorgang bei der Stabilitätsprüfung: Einstellung der Ausgangsposition und Abstandsmessung zwischen Tibiahinterkante und hinterer Begrenzung der Talusrolle (links oben und rechts oben). Dann Stabilitätsprüfung unter Realtime-Bedingungen mit Beobachtung des Auseinanderweichens der Meßpunkte. „Einfrieren" und ausmessen des größten Abstandes (Bildausschnitte links unten und rechts unten).
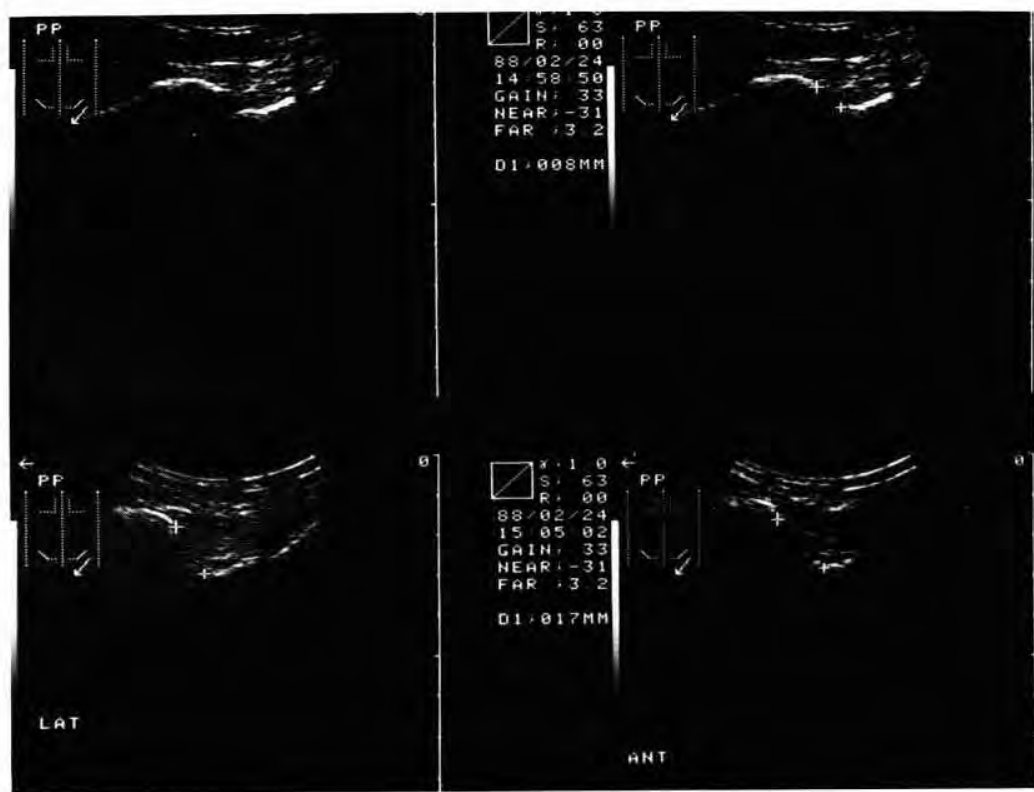

Abb. 5 Beispiel für die Dokumentation einer Sonographie-Untersuchung: Die Standard-Untersuchungsposition wird eingestellt (links oben). Der Talusvorschub wird geprüft (rechts unten), ebenso die Taluskippung (links unten). Die Einhaltung der definierten Untersuchungsebene sollte erkennbar sein. Meßdaten werden vor der Ablichtung eingetragen.

lo-talare anterius eine Sensitivität von 0,96, für die Verletzung des Lig. fibulo-calcaneare eine Sensitivität von 0,79 .

Die gehaltenen Röntgenaufnahmen haben uns im Vergleich zur Sonographie enttäuscht. Wir wollen auf einen Wertevergleich hier nicht eingehen, da sie bei 14 unserer Patienten mit frischen Bandverletzungen trotz exakter Ausführung nicht zur richtigen Diagnose geführt haben. Bei diesen Fällen war die Indikation zur Operation aufgrund des klinischen Befundes gestellt worden. Bei der chronischen Bandinstabilität war bis auf eine Ausnahme eine gute Korrelation zwischen Röntgenaufnahmen und Sonographieergebnis zu sehen.

Nach Abschluß der Diagnostik gaben alle Patienten an, daß die Ultraschalluntersuchung wesentlich weniger schmerzhaft gewesen sei als die gehaltene Röntgenaufnahme. Die Sonographie wurde bis auf seltene Ausnahmen von einem Untersucher ohne Hilfsperson durchgeführt (Zeitaufwand 5 bis maximal 15 Min.).

\section{Diskussion}

Die Ultraschalluntersuchung des oberen Sprunggelenkes sollte sich nicht auf die Stabilitätskontrolle des Außenbandapparates beschränken. Knochen- und Gelenkflächen, Sehnen und Kapsel-Band-Strukturen sollten nach zusätzlichen Verletzungen abgesucht, Hämatome und Hämarthros nach Ausdehnung erfaßt werden. Die unverletzte Gegenseite sollte stets mituntersucht und zum Vergleich herangezogen werden. 
Ultraschalluntersuchungen nach der von Schricker, Hien und Wirth (11) beschriebenen Methode haben gezeigt, daß eine Funktionsdiagnostik des Außenbandapparates des oberen Sprunggelenkes möglich ist (4, 11). In der täglichen Praxis erwiesen sich die dort beschriebenen Schallkopfpositionen bei der Bandprüfung als umständlich und unsicher, so daß wir unsere funktionelle Methode entwickelt haben.

Mit einer Sensitivität von 0,96 für die Verletzung des Lig. fib.-tal, ant. und 0,79 für das Lig. fib.-calc. ist unsere Methode sehr sicher und zeigt Vorteile für das Lig. fib.-tal. ant. gegenüber der Methode der Arbeitsgruppe um Hien (11), deren Sensitivität von Glaser (4) mit 0,906 für das Lig. fib.-tal. ant. und 0,818 für das Lig. fib.-calc. angegeben wird.

Ein Vorteil unserer Sonographie-Methode gegenüber der Methode nach Hien ist, daß die Konturen von Tibiahinterkante und Talusrolle von gehaltenen Röntgenaufnahmen gut bekannt sind und in der Einarbeitungsphase kaum „umgedacht“ werden muß. Das Aufklappen und Klaffen des Gelenkspaltes ist gut zu beobachten. Die Plantarflexion, die bekanntlich einen Talusvorschub vortäuschen kann, bringt die Talusrolle unter der Tibiahinterkante zum Verschwinden und scheidet als mögliche Fehlerquelle aus. Die Methode nach Hien $(4,11)$ beobachtet das Vortreten der Talusrolle vor die Tibiavorderkante, was nicht sicher gegen die Plantarflexion abgegrenzt werden kann. Ein Wechsel der Schallkopfposition für die Prüfung der einzelnen Bänder, sowie eine obligate Hilfsperson bei der Bandprüfung sind bei Hien ebenso von Nachteil (4, 11). Bisher fehlen auch Angaben, ob komplette Rupturen und Teilrupturen der einzelnen Bandanteile zu erkennen sind.

Auch wenn die Taluskippung streng seitlich überprüft und nicht der gesamte Vorfuß supiniert wird, so wie wir es angestrebt haben, bleibt die Beurteilung der Taluskippung problematisch. Die Ruptur des Lig. fib.tal. ant. erlaubt eine Subluxation des Talus nach vorne und in dieser Stellung erschlafft das Lig. fib.-calc. und läßt bereits eine vermehrte Taluskippung zu. Auch die Beurteilung der gehaltenen Röntgenaufnahmen ist aus diesem Grund problematisch. Untersuchungen von Ludolph und Mitarb. (8) haben gezeigt, daß anhand der gehaltenen Röntgenaufnahmen aus Taluskippung und Talusvorschub offensichtlich nicht auf das Verletzungsmuster der Bänder geschlossen werden kann. Bei Sonographie-Methoden hingegen ist dies mit Einschränkung möglich.

Der Wert der Ultraschallmethode muß auch an den etablierten gehaltenen Röntgenaufnahmen gemessen werden. Die Vielzahl von Halteapparaten für die Durchführung von gehaltenen Röntgenaufnahmen, mit vielen Verbesserungsvorschlägen, sogenannte „standardisierte“ Belastung mit Druckwerten zwischen 5 und $25 \mathrm{kp}$, erheblich variierende Meßmethoden und pathologische Werte zeigen, daß offensichtlich keine ideale Lösung gefunden worden ist. Eine Übersicht und weitere kritische Anmerkungen finden sich bei Weiß (12), Zink und Wirth (13). Wenn nur gehaltene Röntgenaufnahmen als ,objektives“ Kriterium für die Instabilität herangezogen werden, so muß man damit rechnen, daß Bandläsionen in einer Größenordnung bis zu $20 \%$ übersehen werden und diese
Methode somit schlechter abschneidet als die Sonographie. Unsere eigene Erfahrung wird diesbezüglich durch eine Vielzahl von Mitteilungen aus der Literatur gestützt (4, 7, $8,9,12,13)$.

Invasive Maßnahmen wie Schmerzausschaltung, Arthrographie und Arthroskopie, die teilweise empfohlen werden $(7,9)$, sind bei den guten Ergebnissen der Sonographie nicht erforderlich. Wenn sich aus der Diagnostik keine Indikation zu einer Operation ergibt, wie z. B. bei einseitiger Bevorzugung der konservativen Therapie $(6,14)$, so sollte auch die invasive Diagnostik abgelehnt werden.

Nachteile der neuen Methode sind unseres Erachtens zum einen die notwendige Einarbeitung. Zum anderen sollte man überlegen, ob zusätzlich zur Sonographie aus forensischen Gründen eine Dokumentation mit gehaltenen Röntgenaufnahmen beibehalten wird, bis die Ultraschallmethode allgemein anerkannt ist.

Die Vorteile dieser neuen Methode sind vorbehaltlich unserer kleinen Fallzahl entscheidend.

Die Ultraschall-Untersuchung ist weit weniger schmerzhaft als die gehaltene Röntgenaufnahme und besonders bei der frischen Bandruptur deshalb auch überlegen (2).

Die Röntgenstrahlenbelastung entfällt und limitiert den Untersuchungsgang nicht.

Die sonographische Funktionsdiagnostik ist eine klinische Kontrolle der Bandstabilität unter „Ultraschall-Durchleuchtung". Dadurch wird aus Synopse von wiederholter klinischer Untersuchung mit Kontrolle des subjektiven Befundes im Ultraschallbild die Diagnose für den Untersucher sehr sicher.

Die Methode hat sich auch in Zweifelsfällen sehr gut bewährt (1).

Die Sonographie könnte in Zukunft die gehaltene Röntgen-Aufnahme ersetzen und nicht zuletzt erhebliche Kosten für die Versicherungsträger einsparen.

\section{Literatur}

${ }^{1}$ Ernst, R., R. Gritzen, A. Weber, S. von Liebe, V. Zumtobel: Sonographie-Diagnostik bei Außenbandrupturen des oberen Sprunggelenkes bei nicht eindeutigen radiologischen Befunden. Langenbecks Arch. Chir. (Kongreßbericht 1988) Suppl. II S. 628

${ }^{2}$ Ernst, R., A. Weber, M. Kemen: Sonographie in der Diagnostik der AuBenbandruptur am oberen Sprunggelenk. Hefte zur Unfallheilkunde 207 (1989) 418-430

${ }^{3}$ Forster, G., G. Scheuba, E. G. Weber: Die standardisierte „gehaltene Aufnahme" zur Diagnostik der Bandverletzungen an der unteren Extremität. Akt. Chirurg. 13 (1979) 239

${ }^{4}$ Glaser, F., W. Friedl, E. Welk: Die Wertigkeit des Ultraschalls in der Diagnostik von Kapselbandverletzungen des oberen Sprunggelenkes. Unfallchirurg 92 (1989) 540-546

5 Godolias, G., H. O. Dustmann: Häufigkeit und Ursachen von Bandverletzungen des Sprunggelenkes bei verschiedenen Sportarten. Orthop. Praxis 21 (1985) 697-702

${ }^{6}$ Jakob, R. P., H. Raemy, R. Steffen, B. Wetz: Zur funktionellen Behandlung des frischen Außenbänderrisses mit der Aircast-Schiene. Orthopäde 15 (1986) 434-440 
${ }^{7}$ Leier, B., H. Hempfling: Frische, isolierte Außenbandverletzungen des oberen Sprunggelenkes - Operationsindikation in Zweifelsfällen durch Arthroskopie. Klinikarzt 12 (1983) 449-456

${ }^{8}$ Ludolph, E., G. Hierholzer, K. Gretenkord: Untersuchungen zur Anatomie und Röntgendiagnostik des fibularen Bandapparates am Sprunggelenk. Unfallchirurg 88 (1985) 245-249

9 Mayer, F., U. Herberger, H. Reuber, U. Meyer: Vergleich der Wertigkeit gehaltener Aufnahmen und der Arthrographie des oberen Sprunggelenkes bei Verletzungen des lateralen Bandkapselapparates. Unfallchirurg 90 (1987) 86-91

${ }^{10}$ Pförringer, $W .:$ Sportartspezifische Weichteilverletzungen von Sprunggelenk und Fuß. Orthop. Praxis 21 (1985) 703710

$"$ Schricker, T., N. M. Hien, C. J. Wirth: Klinische Ergebnisse sonographischer Funktionsuntersuchungen bei Kapselbandläsionen am Knieund Sprunggelenk. Ultraschall 8 (1987) 27-31
${ }^{12}$ Weiß, C.: Die gehaltene Aufnahme des oberen Sprunggelenks - eine einfache Routineuntersuchung? Röntgenpraxis 38 (1985) 385-389

13 Zink, W., C. J. Wirth: Wie sicher ist die apparativ gehaltene Röntgenaufnahme des oberen Sprunggelenkes zur Diagnostik der fibularen Kapselruptur? Orthop. Praxis 21 (1985) 711-717

14 Zwipp, H., H. Tscherne, M. Blauth: Zur konservativen Behandlung der fibularen Bandruptur am oberen Sprunggelenk. Unfallchirurg 88 (1985) 159-167

\section{Dr. med. Rainer Ernst}

Chirurgische Klinik der Ruhr-Universität,

St. Josef-Hospital

Gudrunstr. 56

4630 Bochum 1 